\title{
O papel da atenção primária à saúde no controle de infecções sexualmente transmissíveis em adolescentes
}

\author{
The primary health care role in the control of sexually transmitted infections in teenagers \\ El papel de la atención primaria de salud en el control de las infecciones de transmisión \\ sexual en adolescentes
}

\begin{abstract}
Alícia Thandresse Viana Castro ${ }^{1}$, Igor Eduardo Neves Magalhães ${ }^{1}$, Johnny David Simões Madeira ${ }^{1 *}$, Juliana Garcez Silva Carvalho ${ }^{1}$, Natália Rodrigues Gomes de Assis ${ }^{1}$, Silas César da Silva Ribeiro ${ }^{1}$, Thereza Cristina Gomes Horta².
\end{abstract}

\section{RESUMO}

Objetivo: Analisar o papel da atenção primária à saúde no controle de infecções sexualmente transmissíveis (IST) em adolescentes. Revisão Bibliográfica: Os adolescentes apresentam uma série de comportamentos de risco e vulnerabilidades que os tornam mais susceptíveis a contrair IST. A atenção primária de saúde é um meio imprescindível para divulgação de atividades de prevenção, realizadas diretamente nos centros de saúde ou em parcerias com as escolas. $O$ atendimento do adolescente necessita muitas vezes de busca ativa, devendo ser realizado de modo qualificado e sempre buscando a criação de vínculo e confiança. É importante também que os profissionais de saúde tenham preparo para abordar e atender de forma diferenciada às especificidades de adolescentes homens e mulheres. Considerações Finais: A prevenção é sem dúvidas a melhor forma de controle das IST em adolescentes, portanto, faz-se necessária a criação de políticas públicas voltadas a esse grupo, qualificação dos profissionais de saúde para lidarem com as demandas específicas dessa faixa etária e também, distribuição ampla da principal forma de prevenção, o preservativo.

Palavras chave: Atenção primária à saúde, Adolescente, Doenças sexualmente transmissíveis.

\begin{abstract}
Objective: To analyze the primary health care role in the control of sexually transmitted infections (STI) in teenagers. Bibliographic Review: Teenagers present a series of risky behaviors and vulnerabilities that can make them more susceptible to STI. Primary health care is essential on activities of prevention, made directly inside the basic units or in partnerships with local schools. Appointments for teenagers often require an active search of the individual, so it must be realized in a qualified way and always intending the establishment of bonds and trust. It is also important that health professionals are prepared to approach in different ways according to the specificities of gender. Final Considerations: There is no doubt prevention is the best STI control in teenagers, therefore, it's necessary to create public health politics designed for this particular group, the qualification of health professionals to deal with specific demands of this age and also the large distribution of the best prevention item, condom.
\end{abstract}

Keywords: Primary health care, Adolescent, Sexually transmitted diseases.

${ }^{1}$ Pontifícia Universidade Católica de Minas Gerais (PUC Minas), Betim - MG.

*E-mail: johnny.jdsm@gmail.com

${ }^{2}$ Centro Universitário de Belo Horizonte (UNIBH), Belo Horizonte - MG.

SUBMETIDO EM: 8/2020

ACEITO EM: 9/2020

PUBLICADO EM: 12/2020 


\section{RESUMEN}

Objetivo: Analizar el papel de la atención primaria de salud en el control de las infecciones de transmisión sexual (ITS) en adolescentes. Revisión bibliográfica: Los adolescentes presentan una serie de comportamientos de riesgo y vulnerables que los hacen más susceptibles a ser infectados por ITS. La atención primaria de salud es un medio esencial para difundir las actividades de prevención, llevadas directamente en los centros de salud o en colaboración con los profesionales de los colegios. La atención a los adolescentes requiere una búsqueda activa, y debe tratarse de manera calificada y siempre buscando crear vínculos y confianza entre ellos. También es importante que los profesionales de la salud estén preparados para abordar y atender de manera distintas las necesidades de los adolescentes de ambos géneros. Consideraciones Finales: La prevención es, sin duda, la mejor manera de controlar las ITS en adolescentes, por lo tanto, es necessário crear políticas públicas, capacitando los profesionales de la salud para hacer frente a las demandas específicas de este grupo de edad y también, amplia distribución de la principal forma de prevención, el condón.

Palabras clave: Atención primaria de salud, Adolescente, Enfermedades de transmisión sexual.

\section{INTRODUÇÃO}

O período da adolescência pode ser definido como a transição entre a infância e o início da fase adulta, sendo marcado por mudanças físicas, psíquicas e hormonais, as quais são grandemente influenciadas pelo meio e pelas situações nas quais os jovens estão inseridos. Nesse sentido, a adolescência é um período de bastante mutabilidade, em que cada indivíduo reage de maneira singular em relação a si mesmo e ao meio de convivência (CREMONESE L, et al., 2019; RODRIGUES RP, et al., 2019).

Sabe-se que quando orientados, apoiados e incentivados pela família e pelos profissionais da saúde, estes jovens poderão vivenciar esse período de maneira positiva. Isso pode ocorrer de forma que estes indivíduos conciliam as vivências típicas da adolescência, os cuidados à saúde e também a escolaridade, adquirindo maior consciência acerca de suas ações e as possíveis consequências benignas ou não destas em suas vidas (CREMONESE L, et al., 2019; RODRIGUES RP, et al., 2019).

Assim, devido à fase única em que esses indivíduos se encontram, de profundas mudanças biopsicossociais, os mesmos ficam suscetíveis a diversos agravos à saúde, dentre eles, as infecções sexualmente transmissíveis (IST). As IST são agravos que atualmente consternam os adolescentes e exemplos disso são os dados do boletim epidemiológico de sífilis (2019), onde denota-se um aumento de sífilis adquirida entre adolescentes de 13 a 19 anos, entre os anos de 2017 e 2018 (de 59,1 para 75,8 casos por 100.000 hab.) (BRASIL, 2019).

Sabe-se que a Atenção Primária à Saúde (APS) é a porta entrada do sistema, locus integral e longitudinal em saúde, sendo responsável por coordenar as ações focadas na família e na comunidade. Desse modo, a APS constitui uma parte fundamental do serviço público para o combate das IST e diversos outros agravos, possuindo o papel central na prevenção e promoção à saúde (NASCIMENTO JPR, et al., 2014). Tendo em vista a grande relevância do cuidado específico ao indivíduo adolescente, devido aos fatores intrínsecos próprios dessa faixa etária, o presente estudo busca discutir e elucidar a importância da APS no controle das IST em adolescentes.

\section{REVISÃO BIBLIOGRÁFICA}

\section{Vulnerabilidade dos adolescentes às IST, comportamentos de risco e a influência dos determinantes sociais}

Sabe-se que os determinantes sociais de saúde representam fatores importantes nos processos de saúdedoença dos indivíduos. Dentro dos determinantes estão aqueles não-modificáveis, os modificáveis e até mesmo os macrodeterminantes. Com relação ao último, é possível pensar acerca das condições socioeconômicas e demais fatores culturais e ambientais. 
Segundo Oliveira OS, et al. (2018), tais fatores possuem grande capacidade de aumentar ou diminuir as chances de vulnerabilidades às IST entre os adolescentes. Neste mesmo artigo ainda foram identificados grandes grupos de situações de vulnerabilidade dos adolescentes com relação às IST, entre eles: condições socioeconômicas; início precoce da atividade sexual; falta de uso do preservativo e diferenças de gêneros.

No que tange às condições socioeconômicas, segundo Oliveira PS, et al. (2018), é possível citar a baixa renda e escolaridade, assim como o local de moradia, como fatores que contribuem para a maior vulnerabilidade às IST. Se fazendo importante, dessa forma, a relação entre o meio cultural onde vivem os adolescentes e certos comportamentos de risco, tais como o envolvimento com drogas ou violência familiar e sexual.

O baixo nível econômico caracteriza-se como um determinante, pois entre os adolescentes afetados com IST, uma grande parcela pertence à indivíduos de baixa renda, provenientes de escolas públicas e com maiores dificuldades no acesso às informações adequadas.

Acerca do início precoce da vida sexual, é imperativo o diálogo e fornecimento de informações sobre sexualidade sem tom de crítica, pois, ao passo que muitos dos adolescentes estão imersos culturalmente em um ambiente que incentiva o início sexual precoce, também se nota a falta de informações referentes ao sexo seguro.

De acordo com Amoras BC, et al. (2015), muitos adolescentes iniciam as atividades sexuais antes mesmo dos 15 anos de idade e Costa MIF, et al. (2019) observa que adolescentes do sexo masculino que iniciam a vida sexual antes dos 15 anos, por vezes, o fazem associado a comportamentos de maior risco, como uso de tabaco e álcool. Isso tudo corrobora com a necessidade do diálogo, sendo o ambiente da escola um dos mais adequados para o fornecimento de informações corretas, e, nesse sentido, a boa articulação entre a APS e a escola têm a potência de produzir atividades relevantes em educação em saúde.

No que diz respeito ao uso do preservativo, na revisão de Oliveira PS, et al. (2018) fica evidente os problemas relacionados a sua falta de uso. Além disso, pode-se perceber a desinformação dos adolescentes sobre o tópico em estudo realizado por Cruz LZ, et al. (2018), na Bahia, com 457 jovens entre 16 e 19 anos.

Neste, apesar de 98,9\% dos entrevistados referirem-se à camisinha como método mais eficiente para evitar o contágio com IST, nota-se que existem parcelas relevantes de jovens que acreditam que 0 preservativo pode sumir no corpo da mulher durante a relação sexual ou que o uso de dois preservativos simultaneamente aumenta a chance de prevenção, o que mostra que, apesar da maioria dos indivíduos estarem cientes da necessidade do uso de preservativo, esta informação precisa ser trabalhada com essa faixa etária. Com relação às diferenças de gêneros, este assunto será discutido com mais detalhes posteriormente neste trabalho, devido a sua grande relevância.

Enfim, em face dos determinantes que aumentam os riscos de contaminação com IST pelos adolescentes, é imperativo que a APS e a escola trabalhem em sintonia na construção de informações concretas e na desconstrução dos mitos e tabus que envolvem o tema da sexualidade, pois, conforme constatado por Oliveira PS, et al. (2018), os serviços de saúde são pouco procurados para o fornecimento de informações acerca das IST, sendo que esses jovens preferem se informar com amigos ou familiares. Os motivos apontados para este distanciamento são diversos, entretanto, é papel da APS a identificação da sua população, suas vulnerabilidades e a articulação de políticas voltadas aos adolescentes.

\section{Abordagem respeitosa e escuta qualificada dos adolescentes}

Embora a adolescência seja relatada dentro de uma faixa etária restrita, inclusive pela Organização Mundial de Saúde, conceituá-la cronologicamente dificulta a abrangência de características biopsicossociais. Tais definições de idade apenas facilitam a elaboração de políticas públicas e gerenciamento de ações e estratégias específicas para um público-alvo. Dessa maneira, ressalta-se que os aspectos biopsicossociais precisam ser considerados, sobretudo, na abordagem desses indivíduos (GONDIM PS, et al., 2015). Daí a importância de se ter uma aproximação respeitosa dos adolescentes, com escuta qualificada. 
Uma vez entendido que cada adolescente possui características peculiares, haja visto inclusive que estão expostos a vulnerabilidades diferentes, a saúde e a educação passam a desempenhar um papel de conscientização desse grupo quanto à atenção integral que lhes é de direito. Nesse sentido, é primordial que os profissionais prezem por uma interação que respeite suas culturas e possibilitem uma abertura adequada para concretização de um diálogo eficiente.

Além da família, os profissionais de saúde e de educação devem se consolidar como potenciais elementos de apoio aos jovens. Isso é formidável porque o universo dos adolescentes é repleto de mitos, preconceitos e contradições que precisam ser trabalhadas, dentro de um contexto familiar e social. Ademais, os jovens, apesar de terem acesso aos diversos meios de informações, frequentemente as possuem de maneira equivocada e fora de contexto (HIGA EFR, et al., 2015). Isso, reitera a necessidade de uma abordagem ampliada, que contemple aspectos sociopolíticos e culturais.

Para que se tenha uma forma mais eficiente de relacionamento entre profissionais de saúde e adolescentes, de maneira a estabelecer uma relação horizontal e aberta ao diálogo, deve-se incorporar a ideia desses indivíduos como protagonistas na construção do processo de saúde particular e coletiva. Tais jovens precisam dessa forma de autonomia e emancipação, para se integrarem na responsabilidade social, o que é constatado em estudos como o realizado por Costa ACPJ, et al. (2015), acerca da participação de estudantes na prevenção de IST.

No que se refere ao combate dessas infecções, o trabalho citado inclusive pondera que se deve priorizar a construção de táticas que culminem no autocuidado. Diante das particularidades intrínsecas à adolescência, considerar seus anseios e fazer com que eles se sintam responsáveis por deliberar sobre as questões de sua própria saúde é muito decisivo.

Nesse sentido, a formação de vínculo com os pacientes favorece de maneira especial o diálogo com os adolescentes. A escuta ativa pelos profissionais de saúde, assim como a abordagem respeitosa, sustenta uma conduta de aproximação e possibilita a veiculação de mensagens relacionadas aos conflitos de corpo e mente vivenciados pelos jovens.

Por conseguinte, essas relações de conexão e confiança fazem com que os adolescentes ouçam mais os profissionais e implementem mais as recomendações. Portanto, a escuta do adolescente não pode se resumir a uma escuta usual técnica. Longe disso, ela deve ser um momento para ouvir, orientar, partilhar experiências e buscar soluções em conjunto, incentivando a adoção de escolhas conscientes e responsáveis (COSTA ACPJ, et al., 2015).

Por fim, pode-se afirmar que a abordagem respeitosa e a escuta qualificada são a base do acolhimento e atendimento humanizados. Ações simples, como perguntar ao adolescente como ele prefere ser chamado, e acatar o nome social, quando for o caso, culminam em uma interação sem discriminação, que transparece respeito (BRASIL, 2018). Desse modo, favorece-se a discussão de assuntos diversos, sem julgamentos. Além do mais, não se deve esquecer de enfatizar o caráter sigiloso da consulta para o jovem, para que ele sinta, além de tudo, segurança de partilhar informações e elucidar dúvidas.

Assim, recomenda-se, inclusive, que seja explicado que muitas perguntas são feitas para todos os pacientes, independentemente da idade ou do estado civil (BRASIL, 2020). Dessa forma, é possível estabelecer uma comunicação efetiva com os adolescentes, pautada na humanização e na integralidade da abordagem.

\section{Importância do vínculo entre saúde e escolas}

A educação em saúde nas escolas possui papel importante para conscientização e prevenção de IST, já que estas estão em contato próximo dos adolescentes, grupo etário que apresenta grande vulnerabilidade em adquirir essas patologias. Por isso, é essencial desenvolver nesse público um conhecimento crítico em relação à sua saúde, possibilitando a esses jovens o desenvolvimento do autocuidado e da responsabilidade de se manter saudável e de prevenir as IST, bem como uma gravidez indesejada, a qual pode interferir de modo significativo em seus futuros (AMORAS BC, et al., 2015). 
O Programa Saúde na Escola (PSE) é um importante intermediador entre a saúde e a rede pública de ensino, o qual possui a finalidade de propiciar melhoria da qualidade de vida dos brasileiros por meio de ações de prevenção e promoção à saúde (BRASIL, 2009). Dentre elas, há o cuidado em educar no quesito saúde sexual e reprodutiva de jovens, associando os diversos setores da área da saúde e da própria educação para evitar o contágio de IST, principalmente HIV, e gravidezes indesejadas, as quais são um importante fator de evasão escolar de adolescentes de 10 a 24 anos (COSTA MIF, et al., 2019).

Essas atividades são abrangidas pela Estratégia Saúde da Família (ESF), tornando possível o vínculo entre equipamentos públicos da saúde, como as Unidade Básicas de Saúde (UBS) e as escolas. A área de abrangência é definida de acordo com a territorialização proposta pela ESF e as ações são propostas de acordo com a realidade social e escolar, bem como a capacidade de ação dentre essas áreas de cada local específico. Essas ações integradas, visando a disseminação de informação a partir da associação de duas áreas essenciais, são de grande valor para a conscientização em relação às IST na comunidade (BRASIL, 2009).

Muitas vezes, os adolescentes não procuram a UBS devido à falta de assistência, à má qualificação dos profissionais para lidar com esse público ou ao tabu cultural relacionado à sexualidade. Além disso, grande parte deles não recebem uma orientação sexual dos pais e, por isso, o PSE torna-se uma ponte tão importante entre a saúde e a educação capaz de derrubar conflitos e preconceitos existentes entre os adolescentes, além de proporcionar a disseminação de conhecimento e de conscientização a respeito da própria saúde do indivíduo e de aumentar o vínculo com a APS (AMORAS BC, et al., 2015).

Dessa forma, esses jovens terão informação suficiente para evitarem a contaminação de si mesmos e daqueles com quem se relacionam, podendo ter autoridade sobre o próprio corpo e de suas escolhas, bem como poderão determinar seus futuros, a partir do conhecimento de como evitar uma possível gravidez. Assim, os adolescentes que tiverem acesso a essa gama de informações poderão adquirir maior responsabilidade e irão se conscientizar sobre a importância do uso do preservativo (AMORAS BC, et al., 2015).

Apesar do PSE ser uma ferramenta importante para promoção de saúde pública, é fundamental que sejam criadas outras políticas voltadas exclusivamente para o público jovem, pois a falta delas ressalta a vulnerabilidade existente nessa faixa etária, visto que há poucos programas voltados para a prevenção de IST e de gravidezes indesejadas, fato que auxilia na continuidade desses problemas entre os adolescentes. Dessa forma, se evitará, por exemplo, que um indivíduo assintomático e possível portador de alguma patologia a passe adiante durante uma relação sexual (COSTA MIF, et al., 2019).

\section{O papel do agente comunitário de saúde na prevenção de IST em adolescentes e os desafios encontrados na prática}

O agente comunitário de saúde (ACS) foi incorporado em 1994 ao Programa Saúde da Família (PSF), tornando o ACS parte de uma equipe multiprofissional e extremamente importante para a configuração e efetividade da APS (PEDEBOS LA, et al., 2018). Inicialmente, esperava-se que os ACS fizessem visitas domiciliares às famílias e, se caso detectassem algum problema, encaminhassem a questão aos outros profissionais da equipe (SECCO, AC et al., 2020).

Hoje a Política Nacional de Atenção Básica orienta que cabe aos ACS desenvolver atividades de promoção de saúde, de prevenção das doenças e agravos, por meio de visitas domiciliares e ações educativas individuais e coletivas nos domicílios e comunidade, além de orientação às famílias sobre os serviços de saúde disponíveis no território (BRASIL, 2017).

Entende-se que o ACS exerce papel fundamental no controle de doenças que teriam sua prevalência diminuída caso houvesse prevenção adequada pela população. Um desses grupos de doenças, que é o foco deste artigo, são as IST. Tais infecções, vem aumentado significativamente no grupo etário dos adolescentes. Segundo Oliveira PS, et al. (2018), a maioria das IST no Brasil tem acometido a população de jovens e adolescentes, sendo que a incidência e a prevalência do HIV/AIDS tem aumentado na faixa etária dos 15 aos 24 anos, evidenciando que a prevenção das IST ainda é um desafio para o país. 
A dificuldade de se estabelecer vínculo com a faixa etária dos adolescentes é uma questão importante de saúde e que se baseia numa série de questões específicas desse grupo. Desse modo, a inclusão dos ACS na APS, é indiscutivelmente benéfica pelo fato de serem profissionais que apresentam contato direto com a população de seu território, o que contribui para disseminação de conhecimento e facilidade de acesso à saúde (BRASIL, 2006). Todo o conhecimento desse profissional em relação à comunidade que atende assim como suas observações sobre o que deve ou não ser prioridade para cada família tem grande influência nas ações planejadas pelos profissionais de saúde (SANTOS AO, et al., 2012).

Desse modo, é importante que os ACS tenham a ideia consolidada de que o campo da sexualidade não é menos relevante do que outras necessidade sociais e de saúde que diversas vezes são percebidas como mais urgentes, e que os adolescentes, também devem ser fonte de preocupação, devendo receber a mesma atenção que é dirigida prioritariamente aos pacientes hipertensos, diabéticos, gestantes e bebês (MOROSINI MR e FONSECA AF, 2018). Portanto, cabe ao ACS promover mais diálogo sobre prevenção, através de uma conversa educativa em uma perspectiva não moralista e sim, emancipatória, que construa noção de sujeito cidadão e autonomia.

É papel do ACS organizar grupos de prevenção e convidar a população para participar, além de levar conhecimento até cada família durante as visitas domiciliares, seja conscientizando em relação às próprias IST ou se colocando à disposição quando for necessário. Além disso, esses profissionais conseguem realizar a distribuição do preservativo, um meio acessível, de fácil uso e extremamente importante e eficaz na prevenção de IST (SANTOS AO, et al., 2012).

Entretanto, ter contato mais próximo com a população e acabar criando vínculo e confiança, não criam no profissional a habilidade de conversar e de compartilhar facilmente conhecimentos sobre sexualidade. Em um estudo realizado por Santos AO, et al. (2012), mais de $80 \%$ das agentes entrevistadas disseram não se sentir à vontade para abordar queixas sexuais durante as visitas. Tal fato, chama a atenção para a importância de se aprimorar a formação desses profissionais na área da sexualidade, visto que são assuntos de grande interesse entre os adolescentes, que se bem orientados tem grande potencial de conseguirem prezar por sua saúde e serem protagonistas de suas escolhas.

Os agentes têm grande potencial de ação na promoção e proteção da saúde sexual dos adolescentes e jovens na comunidade, e para tanto necessitam ser instrumentalizados e apoiados pela gestão (MOROSINI MR e FONSECA AF, 2018). Portanto, com uma formação mais qualificada, no campo da promoção da saúde sexual, as equipes de saúde da família teriam ainda mais recursos para contribuir, de modo sustentado, com a prevenção e informação dos adolescentes em relação às IST.

\section{Diferenças de saúde sexual entre os gêneros}

As necessidades dos adolescentes com relação às IST são, por si só, diferentes das necessidades dos adultos. Entre eles, porém, também há diferenças que devem ser tratadas com abordagens variadas. Além das faixas etárias, por exemplo, o sexo e o gênero dos indivíduos deve ser considerado ao estudarmos as melhores formas de prevenção de IST entre esse grupo.

Os agravos em saúde dos adolescentes frequentemente decorrem não de questões puramente biológicas, mas de hábitos e comportamentos, que são em grande medida influenciados pela forma como os jovens são socializados. É preciso que os profissionais de saúde estejam preparados para atender às especificidades de adolescentes homens e mulheres de forma única (BARROS RP, et al., 2019).

Estudo feito por Oliveira PC, et al. (2017) sobre o conhecimento em saúde sexual e reprodutiva dos adolescentes concluiu que as mulheres estavam mais conscientes acerca das IST, dos métodos preventivos existentes e de como obtê-los.

O artigo atribui esse resultado ao fato de que, nas culturas ocidentais, o cuidado com a saúde e as consequências da vivência da sexualidade, como uma possível gravidez e os riscos de IST, são de responsabilidade feminina. Isso está exemplificado no fato de que, entre os alunos entrevistados no estudo, $64,5 \%$ das meninas afirmou usar preservativos em todas as relações sexuais e apenas $40,5 \%$ dos meninos disse o mesmo. 
Outro trabalho, conduzido por Felisbino-Mendes MS, et al. (2018), também aponta para diferenças entre as experiências femininas e masculinas no que diz respeito à vida sexual. Os meninos do estudo iniciaram a vida sexual mais precocemente, apresentaram maior número de parceiros (as), fizeram menos uso de preservativo e receberam menos orientação sobre prevenção de IST e gravidez do que as meninas. Esses dados corroboram os achados de Oliveira PC, et al. (2017) e indicam que os meninos adotam mais comportamentos de riscos, o que justifica uma abordagem diferenciada no trato entre os sexos.

Além disso, há diferenças na facilidade de acesso aos serviços de saúde. Em geral, os rapazes recebem menos atendimento e são pouco incluídos em ações de prevenção, o que mostra uma "invisibilidade" dos meninos pelos profissionais e serviços de saúde. Para as meninas que apresentam sintomas de IST, por exemplo, existem fluxos mais ágeis dentro do SUS e da UBS para atendimento, enquanto os meninos costumam ser encaminhados sem avaliação prévia de um generalista para consulta de especialidade, o que tende a ser mais demorado. Isso corrobora os achados de outros estudos, que mostram que a saúde sexual costuma ser tratada como saúde da mulher, associada a questões do universo feminino (BELLENZANI R, et al., 2012).

Diante desses dados, é inegável que são necessárias políticas públicas diferentes para atender homens e mulheres. É preciso ampliar o acesso à saúde dos homens, criando fluxos de atendimento que facilitem o cuidado. Além disso, são necessárias ações para educar meninos e meninas quanto ao papel fundamental de ambos para evitar IST, ressaltando que o dever de se prevenir não é apenas feminino.

\section{CONSIDERAÇÕES FINAIS}

A partir de todos os dados encontrados, observa-se que o trabalho de prevenção de IST entre adolescentes é uma atividade complexa, que envolve diversos setores, como família, escola e serviços de saúde, e especificidades de cada indivíduo, como sexo, idade, comportamento e condição social. Apesar de difícil, entretanto, é um trabalho extremamente necessário, posto que a prevenção é sem dúvidas a melhor forma de controle das IST. Assim, é necessário que políticas públicas para esse fim sejam constantemente criadas e aprimoradas para garantir o combate efetivo das IST, investindo na qualificação dos profissionais, na educação dos adolescentes e na distribuição ampla do preservativo.

\section{REFERÊNCIAS}

1. AMORAS BC, et al. Reflexões sobre vulnerabilidade dos adolescentes a infecções sexualmente transmissíveis. PRACS: Revista Eletrônica de Humanidades do Curso de Ciências Sociais da UNIFAP. Macapá, 2015; 8(1): 163171.

2. BARROS RP, et al. Adolescente e adolescência: compreensão das necessidades em saúde para atenção integral. CIAIQ2019, 2019; 2: 1627-1636.

3. BELLENZANI R, et al. Agentes comunitárias de saúde e a atenção à saúde sexual e reprodutiva de jovens na estratégia saúde da família. Saúde soc., São Paulo, 2012; 21(3): 637-650.

4. BRASIL. Ministério da Saúde. Saúde na escola. Brasília: Ministério da Saúde, 2009. (Cadernos de Atenção Básica, n. 24) (Série A. Normas e Manuais Técnicos).

5. BRASIL. Ministério da saúde. Secretaria de Vigilância em Saúde. Departamento de Doenças de Condições Crônicas e Infecções. Boletim Epidemiológico de Sífilis (2019). Brasília: Ministério da Saúde, 2019.

6. BRASIL. Ministério da Saúde. Secretaria de Atenção à Saúde. Departamento de Ações Programáticas Estratégicas. Saúde Sexual e Saúde Reprodutiva: os homens como sujeitos de cuidado. Brasília: Ministério da Saúde, 2018.

7. BRASIL. Ministério da Saúde. Secretaria de Atenção À Saúde. Departamento de Atenção Básica. Política Nacional de Atenção. Brasília: Ministério da Saúde, 2017.

8. BRASIL. Ministério da Saúde. Secretaria de Atenção à Saúde. Departamento de Atenção Básica. Política Nacional da Atenção Básica. Brasília: Ministério da Saúde, 2006.

9. BRASIL. Ministério da Saúde. Secretaria de Vigilância em Saúde. Departamento de Doenças de Condições Crônicas e Infecções Sexualmente Transmissíveis. Protocolo Clínico e Diretrizes Terapêuticas para Atenção Integral às Pessoas com Infecções Sexualmente Transmissíveis (IST). Brasília: Ministério da Saúde, 2020.

10. COSTA ACPJ, et al. Protagonismo de adolescentes na prevenção de doenças sexualmente transmissíveis. Acta Paulista de Enfermagem, 2015; 28(5): 482-487.

11. COSTA MIF, et al. Social determinants of health and vulnerabilities to sexually transmitted infections in adolescents. Rev Bras Enferm. 2019; 72(6): 1595-1601. 
12. CREMONESE L, et al. Vivências do Período Gravídico-Puerperal na Perspectiva de Mulheres Adolescentes. Res.: fundam. care. online 2019; 11(5): 1148.

13. CRUZ LZ, et al. Conhecimento dos adolescentes sobre contracepção e infecções sexualmente transmissíveis. Adolesc. Saude, Rio de Janeiro, v Adolescência \& Saúde, 2018; 15(2): 7-18.

14. FELISBINO-MENDES MS, et al. Análise dos indicadores de saúde sexual e reprodutiva de adolescentes brasileiros, 2009, 2012 e 2015. Rev. bras. Epidemiol. São Paulo, 2018; 21(1).

15. GONDIM PS, et al. Acessibilidade dos adolescentes às fontes de informações sobre saúde sexual e reprodutiva. Journal of Human Growth and Development, 2015; 25(1): 50-53.

16. HIGA EFR, et al. A intersetorialidade como estratégia para promoção da saúde sexual e reprodutiva dos adolescentes. Interface - Comunicação, Saúde, Educação, 2015; 19(1): 879-891.

17. MOROSINI MR, FONSECA AF. Os agentes comunitários na Atenção Primária à Saúde no Brasil: inventário de conquistas e desafios. Saúde debate, Rio de Janeiro, 2018; 42: 261-274.

18. NASCIMENTO JPR, et al. Os desafios da institucionalização do ensino superior na modalidade a distância: a visão dos gestores de uma universidade federal. Dissertação (Mestrado em Gestão de Organizações Aprendentes) Centro de Ciências Sociais e Aplicadas. Universidade Federal da Paraíba, João Pessoa, 2014; 129 p.

19. OLIVEIRA PC, et al. Conhecimento em saúde sexual e reprodutiva: estudo transversal com adolescentes. Revista Eletrônica de Enfermagem, 2017; 19(17).

20. OLIVEIRA PS, et al. Vulnerabilidade dos adolescentes às doenças sexualmente transmissíveis na atenção primária. Rev. enferm UFPE online, 2018; 12(3):753-762

21. PEDEBOS LA, et al. A vigilância do território na atenção primária: contribuição do agente comunitário na continuidade do cuidado. Saúde debate, Rio de Janeiro, 2018; 42(119): 940-951.

22. RODRIGUES RP, et al. Estratégias da equipe de Saúde da Família frente os aspectos psicossociais enfrentados pelas adolescentes grávidas. Revista Nursing, 2019; 22(249); 2610-2614.

23. SANTOS AO, et al. Agentes comunitárias de saúde e a atenção à saúde sexual e reprodutiva de jovens na estratégia de saúde da família. Saúde Soc. São Paulo, 2012; 21(3): 637-650.

24. SECCO AC, et al. Educação Permanente em Saúde para Agentes Comunitários: um Projeto de Promoção de Saúde. Gerais, Rev. Interinst. Psicol., Belo Horizonte, 2020; 13(1): 1-17. 